\title{
Rare diseases: Challenges and resources
}

\author{
I saac D. Montoya \\ Rush University, Houston, USA \\ Correspondence: Dr. Isaac D. Montoya, 4210 West Alabama, Houston, Texas 77027. Email: imontoya@affiliatedsyste \\ ms.com
}

Received: October 26, 2011

Accepted: November 6, $2011 \quad$ Published: December 1, 2011

DOI : $10.5430 /$ jnep.v1n1p1

URL: http://dx.doi.org/10.5430/jnep.v1n1p1

\section{Key words}

Nursing, Orphan diseases, Rare diseases

\section{I ntroduction}

Together rare diseases affect millions of people, of all ages, around the world. However, there are countless others who are affected or at risk but remain undiagnosed and unaware of their disease. Rare diseases are frequently serious and life altering while many are life threatening or fatal. By definition rare diseases affect a relatively small population, making it a challenge to develop drugs and medical devices that prevent, diagnose, and treat these diseases. Scientists have found that rare diseases are usually genetic and chronic [1]. EURORDIS has estimated that at least $80 \%$ of rare diseases have genetic origins [2]. They go on to say "other rare diseases are the result of infections and allergies or due to degenerative and proliferative causes.”

The prevalence of a rare disease is only an estimate and changes with location and time. For example, in Canada one in 12 Canadians has a rare disease; however, over 25 million Americans experience a rare disease [3]. In the United States a rare (or orphan) disease is generally considered to have a prevalence of fewer than 200,000 affected individuals. Certain diseases with more than 200,000 affected individuals may be included in this list of rare diseases if certain subpopulations of people who have the disease meet the criteria for prevalence standard for rare diseases [4]. It should be noted that there is not a uniform definition for rare diseases and each country establishes their own definition. In Japan, the legal definition of a rare disease is one that affects fewer than 50,000 patients residing in Japan, or about 1 in 2,500 people [5]. However, the European Commission on Public Health defines rare diseases as "life-threatening or chronically debilitating diseases which are of such low prevalence that special combined efforts are needed to address them"[6]. The term low prevalence is defined as fewer than 1 in 2,000 people. Diseases that are "statistically rare, but not life-threatening, chronically debilitating, or inadequately treated,” are not included in the definition [5].

It is estimated that there are between 6,000-8,000 rare diseases known today affecting millions of persons and causing many patients to wait years for a diagnosis. Consider the following:

- Rare diseases most often are chronic, associated with a disability, and often lead to a premature death.

- Rare diseases are poorly understood due to their complexity and represent a disproportionate portion of health care spending. 
- Patients presenting with a rare disease are frequently misdiagnosed or are undiagnosed due to lack of knowledge of the disease.

- Drug companies are reluctant to conduct research into rare disease treatments because it is challenging to recover the costs of developing drugs and devices for small populations that are at times geographically dispersed.

\section{Rare disease programs}

In 1989, the United States National Commission on Orphan (Rare) Diseases found that clinicians, patients and families all had great difficulties obtaining vital information about the rare disease they were seeking to learn about. The Commission recommended a central depository of information on rare diseases that would facilitate access to information for patients and their families. This depository would draw from existing information systems in the public and private sectors. In 1999, a Special Emphasis Panel at the National Institutes of Health (NIH) on the Coordination of Rare Diseases Research supported the findings of the Commission.

The Rare Diseases Act of 2002 included directions for the Office of Rare Diseases Research (ORDR) to support a centralized clearinghouse for rare diseases information. This was because despite advances having been made through the Internet and the use of various databases, more needed to be done to provide information to clinicians, patients and their families through multiple channels so ORDR at the NIH partnered with the National Human Genome Research Institute (NHGRI, http://www.genome.gov/) and established the Genetic and Rare Diseases Information Center (GARD) in 2002. Using a combination of scientific and medical expertise and resources available at the National Institutes of Health (NIH) in the United States, two goals were established: 1 . To provide answers to patients with mysterious conditions that have long eluded diagnosis; 2. To advance medical knowledge about rare and common diseases.

The program has information on:

- What is known about a genetic or a rare disease;

- What research studies are currently being conducted;

- What genetic testing and genetic services are available;

- Which advocacy groups to contact for a specific genetic or rare disease;

- What has been written recently about a genetic or rare disease in medical journals.

NIH Scientists get their information from:

- $\quad$ NIH resources;

- Medical textbooks;

- Journal articles;

- Web sites;

- Advocacy groups, and their literature and services;

- Clinicians/Nurses and other healthcare providers;

- Medical databases.

By 2010, GARD provided 27,565 direct responses regarding 7,136 rare and/or genetic diseases. The program (GARD) reports receiving approximately 350 requests per month for information and averages 58,797 Website hits per month. People submitting requests come from researchers, physicians, nurses, and other health care professionals as well as from patients and their families. Responses from GARD draw predominantly from existing information sources in the public and private sectors. 
The Information Center provides responses Monday through Friday, noon to 6 PM, EST by telephone, e-mail, letter, or fax in English or in Spanish. General information is regularly posted on the ORDR Web site at http://rarediseases.info. nih.gov/RareDiseaseList.aspx?PageID=1. Disease-specific information as well as general information is available on the GARD home page at http://rarediseases.info.nih.gov/GARD/.

\section{Support for patients and their families}

There are several organizations that offer support to the patients and families affected by a rare disease that clinicians and nurses may find helpful with their patients. The Canadian Organization for Rare Disorders (CORD) is the national network of organizations that represent people affected by rare disorders within Canada. CORD's intention is to provide a strong common voice advocating for a healthcare system and health policy for those with rare disorders. The National Organization for Rare Disorders was established in 1983 by individuals and families with rare diseases and makes available information to anyone around the world. The Genetic Alliance, established in 1986, lists information and support groups for approximately 1200 rare diseases. The Global Genes Project, started by the Children's Rare Disease Network, is creating greater public awareness, supporting the millions of families affected by rare disease through an online community \& collaborative portal and other tools, and are funding innovations to support rare disease research. Patients

with Rare Diseases in Greece are represented by the Greek Alliance of Rare Diseases. This is but a sampling of the organizations offering support in the field of rare diseases.

\section{References}

[1] Aymé S, Schmidtke J. Networking for rare diseases: a necessity for Europe. Bundesgesundheitsblatt esundheitsforschung Gesundheitsschutz. 2007; 50 (12): 1477-83. PMid:18026888 http://dx.doi.org/10.1007/s00103-007-0381-9

[2] EURORDIS: Rare Diseases Europe "What is a rare disease” [Internet]. 2011 October 25. Available from: http://www.eurordis.org/content/what-rare-disease

[3] Canadian Organization for Rare Disorders [Internet]. 2011 October 20. Available from: http://www.raredisorders.ca/aboutUs.html

[4] National Institutes of Health. Rare Diseases and Related Terms. Office of Rare Diseases Research [Internet]. 2011 October 20. Available from: http://rarediseases.info.nih.gov/RareDiseaseList.aspx?PageID=1

[5] Rare Diseases: What are we talking about? [Internet]. 2011 October 24. Available from: http://malattierare.regione.veneto.it/inglese/dicosaparliamo_ing.php

[6] European Commission. Useful Information on Rare Diseases from an EU Perspective [Internet]. 2011 October 23. Available from: http://ec.europa.eu/health/ph_information/documents/ev20040705_rd05_en.pdf 